BIOINFORMATIC APPROACHES FOR THE GENETIC AND PHENOTYPIC CHARACTERIZATION OF A

SACCHAROMYCES CEREVISIAE WINE YEAST COLLECTION

\title{
R. Franco-Duarte ${ }^{1}$, L. Umek ${ }^{2}$, B. Zupan ${ }^{2,3}$, D. Schuller $^{1}$
}

1 Molecular and Environmental Biology Centre (CBMA), Universidade do Minho, Braga, Portugal

2 Faculty of Computer and Information Science, University of Ljubljana, Slovenia
3 Department of Molecular and Human Genetics, Baylor College of Medicine, Houston, Texas, USA

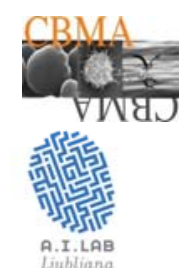

\section{Introduction}
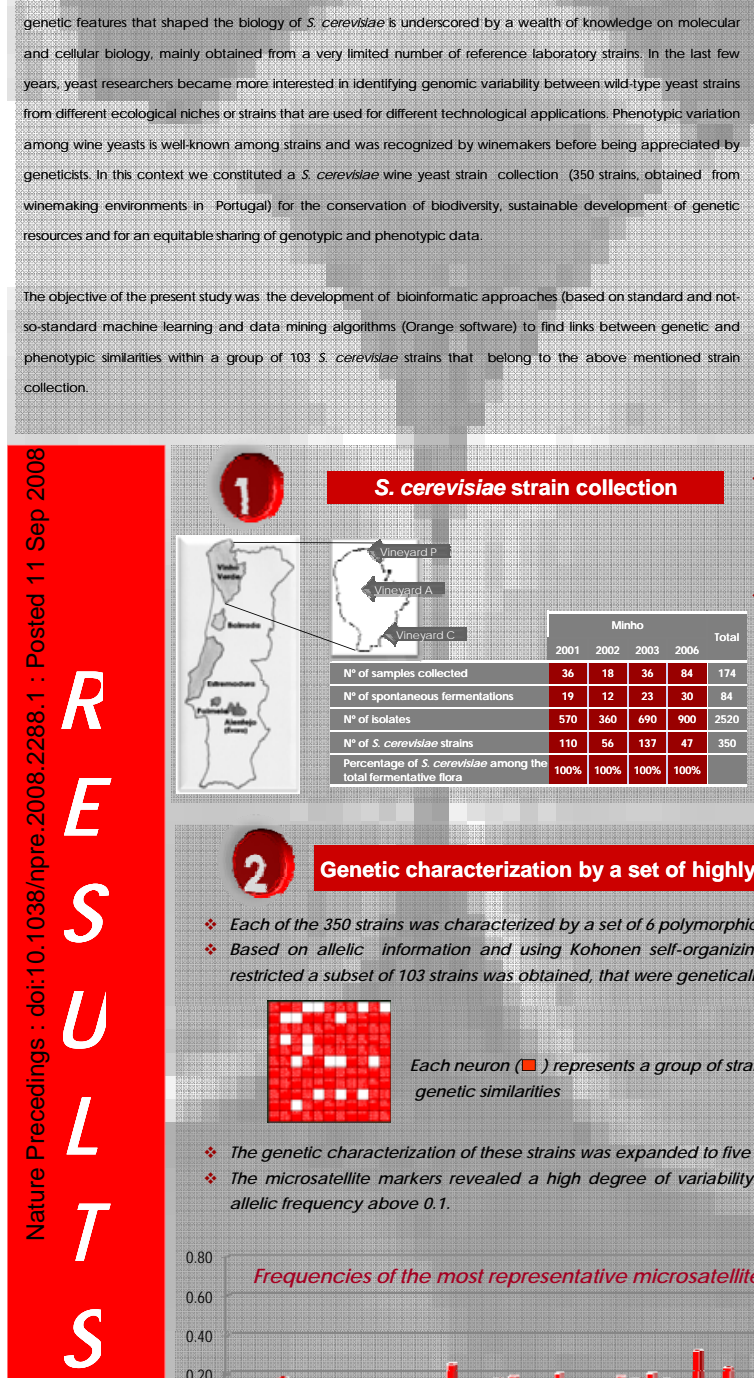

\section{(2)} allelic frequency above 0.1 .

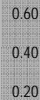

\section{Materials and Methods}

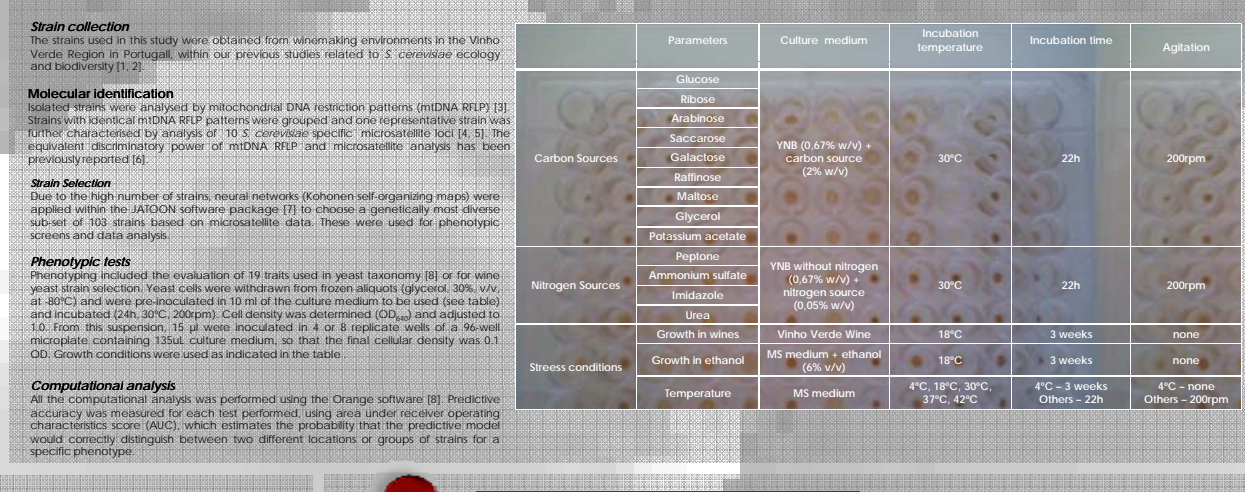

174 grape samples were collected during the havests of fouryears in three vineyards (A, $C$ and $P$ ) of the Vinho Verde Wine Region obtained from the final stages of fementation and $350 \mathrm{~S}$. cerevisiae strains were delimitated, based on mitochondrial DNA restriction fragment length polymorphism analysis * 2520 S. cerevisiae isolates were
Each of the 350 trains was chanacterized by a set of 6 polymorphic microsatilites (SCAATI SCAATE). Based on allelic information and using Kohonen self-organizing maps (JATOON software), a more restricted a subset of 103 strains was obtained, that were genetically most diverse.

$$
\begin{gathered}
\text { Each neuron }(\square) \text { rep } \\
\text { genetic similarities }
\end{gathered}
$$

The genetic characterization of these strains was expanded to five additional microsatellite loci

The microsatellite markers revealed a high degree of variability (171 alleles), and 32 alleles had an

Frequencies of the most representative microsatellites alleles

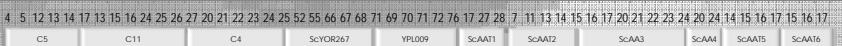

\begin{tabular}{|c|c|c|c|}
\hline \multirow{2}{*}{ Culture medla containing } & \multicolumn{3}{|c|}{ Growth (final $O D_{\text {MaO }}$ ) } \\
\hline & Range & Average & Taxonomy [9] \\
\hline Glucose & $0,9-1,4$ & 1,2 & + \\
\hline Ribose & $0,1-0,6$ & 0,2 & - \\
\hline Arabinose & $0,1-0,5$ & 0,1 & 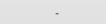 \\
\hline Saccarose & $0,4-1,5$ & 1,1 & $\mathrm{v}$ \\
\hline 5 Galactose & $0,1-1,5$ & 1,0 & v \\
\hline$\frac{8}{\pi}$ Raffinose & $0,2-1,2$ & 0,7 & $\mathrm{v}$ \\
\hline Maltose & $0,2-1,4$ & 1,0 & $\mathrm{v}$ \\
\hline Glycerol & $0,1-0,4$ & 0,2 & $\mathrm{v}$ \\
\hline Pota ssium acetate & $0,1-0,4$ & 0,1 & $\mathrm{v}$ \\
\hline \multirow{3}{*}{ 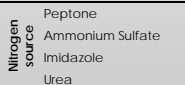 } & $0,6-1,4$ & 1,3 & + \\
\hline & $0,3-1,4$ & 1,0 & $\mathrm{v}$ \\
\hline & $0,2-1,2$ & 0,6 & $\mathrm{v}$ \\
\hline Urea & $0,3-1,4$ & 1,1 & $\mathrm{v}$ \\
\hline $4^{4} 4^{\mathrm{a} C}$ & $0,1-0,3$ & 0,1 & \\
\hline $18^{\mathrm{a} C}$ & $0,2-1,4$ & 1,1 & \\
\hline $30^{\mathrm{a} C}$ & $0,6-1,4$ & 1,0 & + \\
\hline $37^{\circ \mathrm{C}}$ & $0,7-1,5$ & 1,0 & $\mathrm{v}$ \\
\hline $42^{\mathrm{a} C}$ & $0,1-0,3$ & 0,1 & \\
\hline Ethanol $\%(v / v)$ & $0,1-1,3$ & 0,9 & \\
\hline \& Wines & $0,1-0,6$ & 0,1 & \\
\hline
\end{tabular}

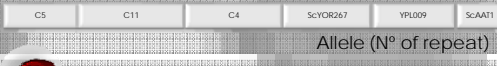

\section{Phenotypic characterization}

Growth in the presence of ethanol $(6 \%, v / v)$

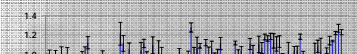

The phenotypic diversity of $103 \mathrm{~S}$. cerevisiae strains was assessed using 22 physiological tests, being 15 used for the conventional identific ation of yeasts

The results generally matched with taxonomic data. However, one and six strains were identified that were capable to consume arabinose and ribose, respectively (O.D. $>0.4)$.

Variation was also apparent for phenotypic traits that are used for wine yeast strain selection such as

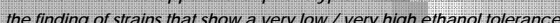

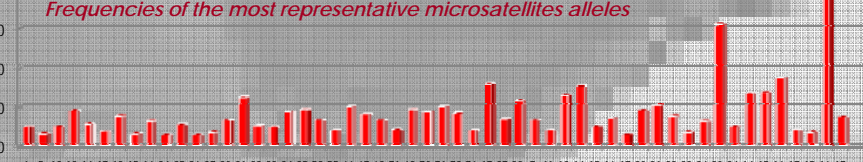

\begin{tabular}{|c|c|c|c|c|}
\hline & \multicolumn{3}{|c|}{ predicted } & \multirow{2}{*}{$\begin{array}{l}\text { Various predic tion methods were tested using } 10 \text {-fold cross validation; best } \\
\text { performance was achieved with naive Bayesian classifier }\end{array}$} \\
\hline \multirow{4}{*}{$\mathrm{a}$} & A & C & $\mathrm{P}$ & \\
\hline & 23 & 4 & 6 & * The table shows a prediction of geographical location (vineyards $A, C$ and \\
\hline & 3 & 14 & 8 & P), based on genetic data (microsatellites). A comect assignment of a strain \\
\hline & 11 & 6 & 28 & to the respective vineyard was obtained \\
\hline \multirow{2}{*}{\multicolumn{4}{|c|}{ lassification accuracy $=0}}$. & \\
\hline & & & & $\begin{array}{l}\text { The area under receiver operating characteristics score (AUC) is high, and } \\
\text { classification accuracy is well above the default accuracy of } 0.45 \text {, which } \\
\text { would be achieved by classifying to the majority class (location P). }\end{array}$ \\
\hline
\end{tabular}

\section{Computational approaches}

Prediction of geographical location Phenotype analysis

One phenotype at the time was analyzed by the Orange software [8], and subgroups were then analyzed through hierarchical clustering. The success of group-characterization was measured through leave-one-out and using AUC score. Subgroups with AUC scores above 0.75 are shown below.
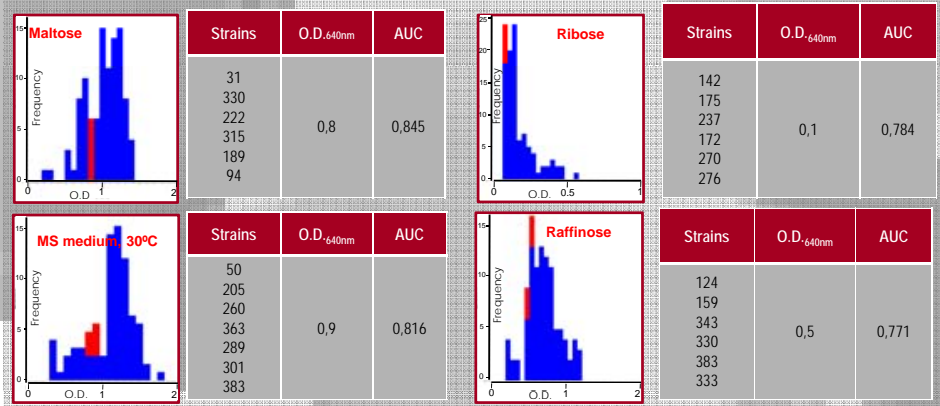

* Tables show strain subgroups with identical or very similar growth rate that also share similarities regarding microsatellite allelic combinations (marked by red bars in the histograms). microsatellite allelic similarities. These strains are genetically not related, they were obtained in different vineyards

* Subgroups of strains with similar growth characteristics in MS medium (average final D.o. $=0.9$ ), YNB containing ribose (average final $D O=0.1$ ) and raffinose (average final $D O=0.5$ ) showed similarities regarding microsatellite allelic pattems

\section{Conclusions}

Strains are genetically variable, demonstrated by the high number (171) of mic rosatellite alleles among 103 strains. This variation is also apparent for phenotypic traits.

Bayesian classifier can assign, with high probability, a strain to the vineyard from where it was isolated.

Hierarchical methods showed that groups of strains that share growth pattems for some culture media (MS medium or YNB containing maltose, ribose or raffinose), can be also grouped based on their mic rosatellite similarities.

Our study shows the potential of computational approaches to obtain indications about strain characteristics from mic rosatellite allelic data.
References
* All 6 strains with intermediate growth in maltose (average final O.D. $=0.8$ ) containing culture medium showed
Acknowledgements

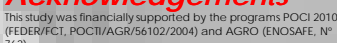

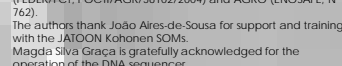

